\title{
In Vitro Release Test Methods for Drug Formulations for Parenteral Applications
}

\author{
Vivian Gray, ${ }^{a}$ Susan Cady, ${ }^{b}$ David Curran, ${ }^{c}$ James DeMuth, ${ }^{d}$ Okponanabofa Eradiri, ${ }^{e}$ Munir Hussain, ${ }^{f}$ \\ Johannes Krämer, ${ }^{\mathrm{g}}$ John Shabushnig, ${ }^{\mathrm{h}}$ Erika Stippler, ${ }^{\mathrm{i}, \mathrm{j}}$ \\ a V. A. Gray Consulting, Inc, Hockessin, DE. \\ b Boehringer Ingelheim Animal Health, North Brunswick, NJ. \\ c GlaxoSmithKline R\&D, King of Prussia, PA. \\ d University of Wisconsin, Madison, WI. \\ e Food and Drug Administration, Silver Spring, MD. -The views presented in this article do not necessarily reflect those of the FDA. No official support or \\ endorsement by the Food and Drug Administration is intended or should be inferred. \\ ${ }^{f}$ Bristol-Myers Squibb Company, New Brunswick, NJ. (Retired). \\ g PHAST, Homburg, Germany. \\ h Insight Pharma Consulting, LLC, Marshall, MI. \\ i United States Pharmacopeia, Rockville, MD. \\ j Correspondence should be addressed to: Desmond G Hunt, Principal Scientific Liaison, US Pharmacopeial Convention, 12601 Twinbrook Parkway, \\ Rockville, MD 20852-1790; tel: +1.301.816.8341; email: dgh@usp.org
}

\section{ABSTRACT}

In vitro drug release testing for parenteral drug formulations could benefit from more regulatory guidance and compendial information as this testing is a part of current expectations for drug product approval. This Stimuli article discusses in vitro drug release methods for those parenteral drug formulations that are not solutions and explores the challenges involved in using these methods for each formulation type.

\section{INTRODUCTION}

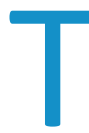

his Stimuliarticle is the work of some members of the General Chapters-Dosage Forms Subcommittee on Parenterals. The Subcommittee is in agreement with and endorses the content of this article.

Parenterally administered drugs include both injected and implanted drug formulations. While injections are administered through the external boundary tissue, implants are placed within the body to allow direct administration of the drug substance(s) into the circulatory system or the local area. Injections exist as solutions, suspensions, or suspensions with a modifiedrelease component. Immediately prior to administration, the drug formulations may be reconstituted from sterile powders to form solutions, suspensions, and emulsions. This category also includes drug-and-device combinations such as stents.

The definitions and descriptions of these dosage forms, as well as brief information about their composition and manufacturing processes, are found in Pharmaceutical Dosage Forms $\langle 1151\rangle$ (1).

In the case of parenteral (non-solution) drug formulations, the purpose of both the product quality tests and product performance tests is to provide assurance of batch-tobatch quality, reproducibility, reliability, and performance. Product quality tests are performed to assess attributes such as assay, identification, impurities, foreign and particulate matter, sterility, bacterial endotoxins, water content, aluminum content, and content uniformity. These tests are part of the compendial monograph [see Injections and Implanted Drug Products $\langle 1\rangle$ (2)].

In vitro drug release testing for parenterals that are nonsolutions could benefit from more regulatory guidance and compendial information. Method selection can be challenging for many reasons, and each formulation carries its own unique obstacles. The Dissolution Procedure: Development and Validation 〈1092〉 (3) is an excellent reference for use when developing an in vitro release test procedure.

Each type of parenteral formulation is discussed in this Stimuli article, including highlights of the formulation's unique challenges. Examples of methods for current formulations are provided; these examples were obtained from multiple sources, including but not limited to the FDA Dissolution Method Database (4), workshop reports $(5,6)$, previous Pharmacopeial Forum Stimuli articles $(7,8)$, and the literature. For several parenteral drug formulations, procedures described in Dissolution $\langle 711\rangle$ (9), Drug Release $\langle 724\rangle$ (10), Mucosal Drug Products-Performance Tests 〈1004〉 (11), or Semisolid Drug Products-Performance Tests $\langle 1724\rangle$ (12) may be applicable.

Because of the complex nature of some parenterals, the in vitro drug release test methods will be applied on a case-by-case basis rather than using a one-size-fits-all 
approach; however, the inclusion of general approaches and the presentation of options will be useful. Implanted parenteral drug formulations are long-acting dosage forms that provide continuous release of the drug substance(s), often for weeks, months, or years. For systemic delivery, these dosage forms may be placed subcutaneously; for local delivery, they may be placed in a specific region of the body. The in vitro test conditions should mimic specific aspects of the intended physiological environment, such as the osmolarity, $\mathrm{pH}$, or buffer capacity. Non-sink conditions may be informative in some instances.

The drug release test for some parenteral dosage forms may require the use of modified compendial or noncompendial equipment. For example, various volumes of dissolution medium with or without agitation may be appropriate. The use of dialysis membrane has been shown to be beneficial for some microsphere injectable formulations. Incubation methods have also been used. Accelerated testing is a practical solution for quality control testing; however, it should be justified as having relevance to "real time" release.

Although desirable, in vitro and in vivo correlations may not be possible for modified-release parenteral dosage forms due to the complexity of the release mechanisms and the lack of knowledge about in vivo release conditions.

\section{SUSPENSIONS}

Although liposomes, microspheres, and nanosuspensions are considered suspensions, the discussion in this section is limited to formulations of poorly water-soluble drug substances or drug substances formulated with a releasecontrolling component, which is typically suspended in an aqueous medium.

\section{Nanosuspensions}

In cases where suspensions exhibit, in principle, particle sizes between 1 and $100 \mathrm{~nm}$, they are called nanosuspensions and have a tolerance extension up to $1000 \mathrm{~nm}$ (13). This suggests that dilution of the suspension prior to dissolution testing may alter the inherent drug release and/or dissolution characteristics significantly.

The most relevant parameters to modify for in vitro performance testing are the content of organic solvents, the surfactants, and the $\mathrm{pH}$ of the media. The physiological range for injectable dosage forms is around $\mathrm{pH} 7.4$ and is narrower than that for oral dosage forms, even when pathophysiological conditions are considered. In the case of osmotic systems, the osmolality of the in vitro drug release media composition requires attention. Diffusion mechanisms may prevail in the case of embedded or coated nanoparticles (14).
The U.S. Food and Drug Administration (FDA) is providing guidance on in vitro performance testing of either modified-release parenteral formulations or drug formulations containing nanomaterials (13). In vitro drug release testing should be based on the compendial instruments described in $\langle 711\rangle(9)$ and $\langle 724\rangle(10)$. The most critical steps of in vitro testing are considered to be the insertion of the suspension into the vessel or cell and the phase separation after sampling. For filtration, membrane filters are preferred. Cross-flow filtration has been shown to be advantageous (15). Compendial systems such as the basket or paddle and the flow-through cell apparatus allow the insertion of the suspension in a bag, which often consists of dialysis membranes with a defined molecular cut-off. Alternatively, reversed dialysis techniques are applied with the suspension spread in the dissolution bulk medium.

The use of in situ fiber optics combined with derivative spectroscopy has been shown to allow the quantification of the dissolved drug substance in the presence of the nanoparticle-bound moiety of the active ingredient (16).

The composition of the drug-release medium may be kept constant with regard to the physiological parameters for $\mathrm{pH}(\mathrm{pH}$ 7.4) and osmotic pressure $(285 \mathrm{mOsm} / \mathrm{kg}$ ). Because of the solubility of the drug substance and the requirement for an accelerated test, the addition of surfactants [e.g., Tween 80 or sodium lauryl sulfate (SLS)] and/or organic solvents may be needed. Accelerated tests are highly desirable. In addition to in vitro drug-release testing, data on the biological activity of the drug product may be needed for proper in vitro performance testing, particularly if the active ingredient is a biological, or in the case of targeting and the route of administration.

\section{Liposomes}

Liposomes are microvesicles composed of a lipid bilayer, and/or a concentric series of multiple bilayers, separated by aqueous compartments. The compartments are formed by amphipathic molecules, such as phospholipids, that enclose a central aqueous compartment. Generally, liposomal formulations comprise the drug substance and lipids, as well as nonlipid and nonliposome inactive ingredients. In liposomal drug formulations, the drug substance is encapsulated within the aqueous compartment(s) or intercalated within the lipid bilayer(s).

The release of drugs from liposomal formulations can be modified by the presence of polyethylene glycol (PEG) and/or cholesterol or other potential additives. It is pertinent to mention that the presence of PEG on the surface of the liposomal carrier has been shown to 
extend blood-circulation time, leading to prolonged drug release in vivo. Although in vitro drug release is one of the critical quality attributes (CQAs) that is useful for characterizing liposomal drug formulations, and extensive research has been done on the technology since the 1980s, development of discriminating and robust in vitro drug release tests for liposomal delivery systems has not become universal.

The lack of standard equipment and commonly available drug release testing methods for liposomal drug formulations is evident when compared to other dosage forms and drug delivery systems. Two dissolutionmethods databases, one from FDA and one from USP, do not contain in vitro drug release tests for liposomes. There is, however, FDA guidance on chemistry, manufacturing, and controls (CMC) aspects of liposomes (17). At present, developers of liposomal drug formulations have proprietary in vitro drug release methods for use in the quality control of their formulations. More collaborative efforts among academia, industry, and regulators are needed to standardize in vitro drug release testing of liposomes (18).

The characteristics of an in vitro drug release test for liposomal drug formulations include, but are not limited to, the following:
1. Mimics the drug release conditions in vivo

2. Discriminates between liposomal formulations of various different compositions

3. Demonstrates the absence of drug release before reaching the target tissue or organ

4. Is based on the intended application of the formulation

5. Reflects the route of administration to establish a reliable in vitro in vivo correlation (IVIVC) during drug development

It is well known that the in vitro release (including dissolution) of a drug from a drug product is formulation dependent. Therefore, it is not possible to design a universal in vitro release method that can be used to characterize drug release from different types of liposomes. Assessing the suitability of a method for a liposomal drug product is therefore crucial. The more common drug release methods reported in the literature for liposomes, and their associated equipment (or apparatus), are summarized in Table 1 (19).

Table 1. Common Drug Release Methods for Liposomes and Associated Equipment

\begin{tabular}{|c|c|c|c|c|c|}
\hline Method & Release Time & $\begin{array}{l}\text { Liposome } \\
\text { Damage }\end{array}$ & $\begin{array}{l}\text { Continuous } \\
\text { Sampling }\end{array}$ & Unique Advantages & Limitations \\
\hline Dialysis & Rapid and Slow & No & Yes & Simple/economic & Sink conditions/membrane dependent \\
\hline Fractional dialysis & Rapid and Slow & No & Yes & Perfect sink conditions & Further validation needed \\
\hline Reverse dialysis & Rapid and Slow & No & $\begin{array}{l}\text { Yes/multiple } \\
\text { sacs }\end{array}$ & $\begin{array}{l}\text { Simple/long sampling time/ } \\
\text { economic }\end{array}$ & Rate-limiting membrane \\
\hline Microdialysis & Rapid and Slow & No & Yes & Minimal manual handling & Drug dependent \\
\hline Ultracentrifugation & Slow & Yes & No & Drug dependent & Sedimentation rates of components \\
\hline Centrifugal ultrafiltration & Slow & Yes & No & Low centrifugal force & Filter clogging/ particle deformation \\
\hline Pressure ultrafiltration & Rapid and Slow & Yes & No & $\begin{array}{l}\text { Gentle separation at low } \\
\text { pressure }\end{array}$ & Filter clogging/ particle deformation \\
\hline $\begin{array}{l}\text { Size exclusion } \\
\text { chromatography }\end{array}$ & Rapid and Slow & No & Yes & Filter media selection & Column pre-saturation needed \\
\hline In situ method & Rapid and Slow & No & Yes & Direct measurement & Polarography and UV/V is limited \\
\hline Continuous flow method & Rapid and Slow & No & No/Yes & Measures instantaneous release & Filter clogging/ physical limitations \\
\hline USP Apparatus IV & Rapid and Slow & No & Yes & Long sampling time & High volume of release medium \\
\hline USP Apparatus I & Rapid and Slow & No & Yes & $\begin{array}{l}\text { Constant surface area for } \\
\text { release }\end{array}$ & Two-stage diffusion \\
\hline
\end{tabular}


It is advisable to consult with the appropriate regulatory agency to confirm the adequacy of an in-house in vitro drug release test for a liposomal drug product before filing the application for approval.

\section{Microparticles (Microspheres)}

This category includes injections containing extendedrelease particles, which are particulates, microparticulates, or microspheres. There are two distinct challenges when performing an in vitro drug release test on this dosage form. First, the particles are likely to float when introduced to the media. Therefore, the mixing would not be uniform, as some of the particulates would be floating on the media surface. Also, these dosage forms may require a prolonged testing time, which could cause extensive evaporation of the dissolution medium. The chemical stability of the dissolved drug in the medium is also a consideration.

When considering an apparatus for injections, the FDA database (4) currently includes 15 injections, and methods are provided for 9 injectable suspensions. In the database, when a specific method is not given, the instructions state, "Develop a dissolution method using USP IV (FlowThrough Cell), and, if applicable, Apparatus II (Paddle) or any other appropriate method, for comparative evaluation by the Agency." In the 9 injectable suspensions that have a method provided, the apparatus are USP Apparatus 4 and 2, and the media include components such as SLS, polysorbate, saline, sodium azide, and water, with a small amount of ethanol. The time ranges extend in duration from $45 \mathrm{~min}$ to as long as $168 \mathrm{~h}$. Methods that use dialysis membranes have been reported in the literature (20), and incubation methods (e.g., Goserelin implants, USP) (21) have been reported as approved regulatory methods.

\section{Emulsions}

For emulsions, the drug substance is typically trapped within a water-in-oil or oil-in-water dispersed phase. These parenteral formulations require less complex in vitro performance tests. Typically, the apparatus described in $\langle 711\rangle$ (9), $\langle 1724\rangle$ (12), and $\langle 1004\rangle$ (11) may be appropriate; these apparatus include USP Apparatus 2, a vertical diffusion cell, and reduced-volume equipment (e.g., a mini paddle).

\section{IMPLANTS}

For long-acting implants in particular, the in vitro drug performance test should include a procedure to ascertain that the drug releases as intended and to prove the robustness of the drug product. There may be real-time (long-term) drug release tests used along with accelerated drug release tests. Some accelerated strategies include elevated temperatures or non-neutral $\mathrm{pH}$ to increase the hydrolysis of rate-controlling polymers. When longterm tests are needed, special attention should be paid to the evaporation of the dissolution media and bacterial growth. In the USP monograph Goserelin Implants, the apparatus is a sealed jar that is incubated (21). Apparatus 4 and Apparatus 7 are also used (5-8). The FDA database provides three implant methods: the Goserelin Implant uses an incubation method, the Dexamethasone Implant uses Apparatus 7, and the Buprenorphine $\mathrm{HCl}$ Implant uses Apparatus 2.

\section{DRUG-ELUTING STENTS}

Drug-eluting stents (DES) are a combination of a device and a drug, where the drug is usually within a polymeric matrix that coats a metal stent. As with implants and controlled-release microparticle suspensions, the longacting nature of the DES makes long tests necessary to fully characterize the release. Long tests come with the challenges of media evaporation and stability of drug substances in the media. The possibility of accelerated conditions is an important consideration, as the in vivo release may occur over a long period of time. Other considerations are the positioning of the DES in the apparatus for adequate mixing and the use of a small volume of medium due to the low drug concentration.

There are in vitro performance test methods suggesting the use of a reduced-volume paddle apparatus, USP Apparatus 4, or USP Apparatus 7 with stent holders, where small volumes of media are used (7). For the approved DES, sirolimus, USP Apparatus 4 (flow rate of $25 \mathrm{~mL} / \mathrm{min}$ ) was used with an elution medium $(50 \mathrm{~mL})$ of $2 \% \mathrm{SLS}$ and $10 \%$ acetonitrile at $\mathrm{pH} 4.5,37^{\circ} \mathrm{C}$ (22). This is a closed-loop configuration.

The method needs to reflect the transport forces that are predominant in vivo (23). This has been demonstrated in methods using a blood vessel-simulating flow-through cell apparatus (24). Other apparatus that have been used for in vitro performance testing of DES are the incubation cells of different volumes and agitation from 250 to 300 rpm at $37{ }^{\circ} \mathrm{C}$, or USP Apparatus 7 with small-volume reciprocating holders in 10-mL glass cells at dip rates of 5 DPM or 40 DPM (25). 
Table 2. Possible Apparatus for In Vitro Release Testing

Procedures for Parenteral Formulations

\begin{tabular}{|l|l|}
\hline \multicolumn{1}{|c|}{$\begin{array}{l}\text { General Chapter } \text { Heading } \\
\text { Apparatus }\end{array}$} \\
\hline Solutions & None \\
\hline Aqueous & Apparatus 2 \\
\hline Oils & None \\
\hline $\begin{array}{l}\text { Powders (for } \\
\text { Solution) }\end{array}$ & $\begin{array}{l}\text { Apparatus 2, Apparatus 4, reduced volume } \\
\text { apparatus, dialysis cell }\end{array}$ \\
\hline Suspensions & Apparatus 2, Apparatus 4, dialysis cell \\
\hline Nanosuspensions & $\begin{array}{l}\text { Apparatus 1, Apparatus 2, Apparatus 4, } \\
\text { dialysis cell }\end{array}$ \\
\hline Liposomes & $\begin{array}{l}\text { Apparatus 2, Apparatus 4, incubation jar, } \\
\text { dialysis cell }\end{array}$ \\
\hline $\begin{array}{l}\text { Microparticles } \\
\text { (microspheres) }\end{array}$ & See Suspensions \\
\hline $\begin{array}{l}\text { Sterile Powders for } \\
\text { Suspension }\end{array}$ & $\begin{array}{l}\text { Apparatus 2, reduced volume apparatus, } \\
\text { vertical diffusion cell }\end{array}$ \\
\hline Emulsions & $\begin{array}{l}\text { Apparatus 2, Apparatus 4, incubation jar, } \\
\text { Apparatus 7 }\end{array}$ \\
\hline Implants & $\begin{array}{l}\text { Apparatus 7, Apparatus 4, Modified flow } \\
\text { through cell }\end{array}$ \\
\hline Drug-eluting stents & See Implants \\
\hline In-situ forming & \\
\hline
\end{tabular}

\section{VETERINARY PARENTERALS}

There are many different types of parenteral formulations commercialized in the veterinary industry. FDA Center for Veterinary Medicine (CVM) Guidance for Industry \#238, issued in June 2016, provides guidance and strategies for consideration as part of the development program for drug release testing and product specifications (26). The drug release test method should be developed early in product development so it can be used to characterize the formulations utilized in safety and efficacy trials. The drug release data for the batches used in these studies support the specifications for the drug release test at product release and expiry. The guidance provided by formulation type in this article should also be considered for veterinary formulations. One recent example reported by Folger et al. describes the work conducted for a veterinary drug product consisting of a lipophilic drug dissolved in a low-viscosity oily matrix (27). This work included the identification of a suitable medium, which dissolved the drug over time without degrading it, and the development of a system and methodology that could be used in the quality control lab and could discriminate a quality product from one that was formulated or processed incorrectly.
Some of the dosage forms may be physically larger than conventional human-sized drug formulations, requiring modifications of the laboratory equipment. Some of these veterinary formulations are designed to give season-long control, releasing the drug for extended periods of time. It is important to coordinate the in vitro drug release method strategy for product development with the quality control (QC) method for release of the product. This strategy is useful in product lifecycle management to support possible manufacturing changes.

\section{CONCLUSIONS}

While the need to demonstrate in vitro performance is recognized by regulatory agencies, industry, and academia, there are currently no compendial or regulatory guidances. The USP Subcommittee on Parenterals is interested in receiving feedback on this Stimuli article to help develop a companion informational general chapter on in vitro release of parenteral drug formulations. Please send comments to Desmond Hunt, dgh@usp.org.

\section{CONFLICT OF INTEREST}

The authors did not declare any perceived or actual conflicts of interest related to the subject matter of this Stimuli article. The views presented in this article do not necessarily reflect those of the organizations for which the authors work. No official support or endorsement by these organizations is intended or should be inferred.

\section{REFERENCES}

1. USP. Pharmaceutical Dosage Forms 〈1151). In: USP 41-NF 36. Rockville, MD: USP; 2017:7425-7450.

2. USP. Injections and Implanted Drug Products (Parenterals)Product Quality Tests 〈1〉. In: USP 41-NF 36. Rockville, MD: USP; 2017:5915-5921.

3. USP. The Dissolution Procedure: Development and Validation 〈1092〉. In: USP 41-NF 36. Rockville, MD: USP; 2017:7178-7198.

4. Food and Drug Administration. Dissolution Methods Database. https://www.accessdata.fda.gov/scripts/cder/dissolution/index. cfm. Accessed 22 May 2018.

5. Burgess $D$, Hussain A, Ingallinera $T$, Chen M. Assuring quality and performance of sustained and controlled released parenter als: AAPS Workshop Report, Co-Sponsored by FDA and USP. Pharm Res. 2002; 19(11):1761-1768.

6. Brown C, Friedel H, Barker A, Bushe L, Keitel S, Cecil T, et al. FIP/ AAPS joint workshop report: Dissolution/in vitro release of nov el/special dosage forms. AAPS PharmSciTech. 2011; 12(2):782794.

7. Shah V, DeMuth J, Hunt D. Performance tests for parenteral dos age forms. Pharm Forum. 2015; 41(3). 
8. Burgess D, Clarke B, Hampson-Carlin M, Shah P. Critical quality and performance parameters for modified-release parenteral dosage forms. Pharm Forum. 2005; 31(6).

9. USP. Dissolution 〈711〉. In:USP 41-NF 36. Rockville, MD: USP; 2017: 6459-6469.

10. USP. Drug Release 〈724〉. In:USP 41-NF 36. Rockville, MD: USP; 2017: 6471-6478.

11. USP. Mucosal Drug Products-Performance Tests $\langle 1004\rangle$. In: USP 41-NF 36. Rockville, MD: USP; 2017: 6699-6702.

12. USP. Semisolid Drug Products-Performance Tests 〈1724〉. In: USP 41-NF 36. Rockville, MD: USP; 2017: 7944-7956.

13. Food and Drug Administration. Draft guidance for industry: Drug products, including biological products, that contain nanomaterials. December 2017.

14. Guillot A, Limberger M, Kramer J, Lehr C-M. In situ drug release monitoring with a fiber-optic system: Overcoming matrix interferences using derivative spectrophotometry. Dissolut Tech nol. 2013; 20(2):15-19.

15. Schichtel J. Dissertation. Determination of the Dissolution Behavior of Celecoxib-Eudragit E 100-Nanoparticles using CrossFlow Filtration. Johannes Gutenberg-Universität Mainz, 2016.

16. Türeli AD. Dissertation. Nanoparticle Preparation Process Using Novel Microjet Reactor Technology for Enhancing Dissolution Rates of Poorly Water-Soluble Drugs. Johannes GutenbergUniversität Mainz, 2005.

17. Food and Drug Administration. Guidance for industry: liposomal drug products-chemistry, manufacturing, and controls; human pharmacokinetics and bioavailability; and labeling documentation. April 2018.

18. Immordino ML, Dosio F, Cattel L. Stealth liposomes: review of the basic science, rationale, and clinical applications, existing and potential. Int J Nanomedicine. 2006; 1(3):297-315.
19. Solomon D, Gupta N, Mulla NS, Shukla S, Guerrero YA, Gupta V. Role of in vitro release methods in liposomal formula tion development: challenges and regulatory perspective. AAPS J. 2017; 19(6):1669-1681.

20. Kostanski J, Deluca P. A novel in vitro release technique for peptide-containing biodegradable microspheres. AAPS Pharm Sci. 2000; 1(1):30-40.

21. USP. Goserelin Implants. In: USP 41-NF 36. Rockville, MD: USP; 2017:1985-1989.

22. Merciadez M, Mehta A, Patel A, Wang A. A novel method for the elution of sirolimus (Rapamycin) in drug eluting stents. Dissolut Technol. 2011; 18(4):37-42.

23. Seidlitz A, Weitschies W. In vitro dissolution methods for controlled release parenterals and their applicability to drug eluting stent testing. J Pharm Pharmacol. 2012; 64:969-985.

24. Seidlitz A, Nagel S, Semmling B, Grabow N, Sternberg K, Weitschies W. Biorelevant dissolution testing of drug eluting stents. Experiences with a modified flow-through cell setup. Dissolut Technol. 2011; 18(4):26-34.

25. Seidlitz A, Schick W, Reske T, Senz V, Grabow N, Peterson S, et al. In vitro study of sirolimus release from a drug-eluting stent: Comparison of the release profiles obtained using different test setups. Eur J Pharm Biopharm. 2015; 93:328-338.

26. US Food and Drug Administration, Center for Veterinary Medicine. Guidance for industry \#238. Modified release veterinary parenteral dosage forms: Development, evaluation and establishment of specifications. June 2016. https://www.fda. gov/downloads/AnimalVeterinary/GuidanceComplianceEnforce ment/GuidanceforIndustry/UCM481721.pdf. Accessed May 22, 2018

27. Priddy TS, Roush RR, Bryson L. and Folger M. Characterization of the in vitro drug exchange profile of a modified-release parenteral solution for veterinary use. Dissolut Technol. 2017; 24(1):6-12. 